Annales Academiæ Scientiarum Fennicæ

Mathematica

Volumen 40, 2015, 215-226

\title{
THE MAXIMAL BEURLING TRANSFORM ASSOCIATED WITH SQUARES
}

\author{
Anna Bosch-Camós, Joan Mateu and Joan Orobitg \\ Universitat Autònoma de Barcelona, Departament de Matemàtiques \\ 08193, Bellaterra, Barcelona, Catalonia; annaboschcamos@gmail.com \\ Universitat Autònoma de Barcelona, Departament de Matemàtiques \\ 08193, Bellaterra, Barcelona, Catalonia; mateu@mat.uab.cat \\ Universitat Autònoma de Barcelona, Departament de Matemàtiques \\ 08193, Bellaterra, Barcelona, Catalonia; orobitg@mat.uab.cat
}

\begin{abstract}
It is known that the improved Cotlar's inequality $B^{*} f(z) \leq C M(B f)(z), z \in \mathbf{C}$, holds for the Beurling transform $B$, the maximal Beurling transform $B^{*} f(z)=\sup _{\varepsilon>0} \mid \int_{|w|>\varepsilon} f(z-$ w) $\frac{1}{w^{2}} d w \mid, z \in \mathbf{C}$, and the Hardy-Littlewood maximal operator $M$. In this note we consider the maximal Beurling transform associated with squares, namely, $B_{S}^{*} f(z)=\sup _{\varepsilon>0} \mid \int_{w \notin Q(0, \varepsilon)} f(z-$ $w) \frac{1}{w^{2}} d w \mid, z \in \mathbf{C}, Q(0, \varepsilon)$ being the square with sides parallel to the coordinate axis of side length $\varepsilon$. We prove that $B_{S}^{*} f(z) \leq C M^{2}(B f)(z), z \in \mathbf{C}$, where $M^{2}=M \circ M$ is the iteration of the Hardy-Littlewood maximal operator, and that $M^{2}$ cannot be replaced by $M$.
\end{abstract}

\section{Introduction}

Although we only work with the Beurling transform and its iterations, we will initially consider more general Calderón-Zygmund singular operators. We begin by introducing the notation and some well known results. Let $T$ be a smooth homogeneous Calderón-Zygmund singular integral operator on $\mathbf{R}^{n}$ with kernel

$$
K(x)=\frac{\Omega(x)}{|x|^{n}}, \quad x \in \mathbf{R}^{n} \backslash\{0\},
$$

where $\Omega$ is a homogeneous function of degree 0 whose restriction to the unit sphere $S^{n-1}$ is $C^{\infty}$ and satisfies the cancellation property

$$
\int_{|x|=1} \Omega(x) d \sigma(x)=0
$$

$\sigma$ being the normalized surface measure in $S^{n-1}$. Let $T f$ be the principal value convolution operator

$$
T f(x)=\text { p.v. } \int f(x-y) K(y) d y \equiv \lim _{\varepsilon \rightarrow 0} T^{\varepsilon} f(x),
$$

where $T^{\varepsilon} f$ is the truncated operator at level $\varepsilon$ defined by

$$
T^{\varepsilon} f(x)=\int_{|y|>\varepsilon} f(x-y) K(y) d y=\int_{|x-y|>\varepsilon} f(y) K(x-y) d y .
$$

doi:10.5186/aasfm.2015.4016

2010 Mathematics Subject Classification: Primary 42B20, 42B25.

Key words: Cotlar's inequality, maximal Beurling transform, Calderón-Zygmund operators. 
For $f \in L^{p}, 1 \leq p<\infty$, the limit in (2) exists for almost all $x$. One says that the operator $T$ is even (or odd) if the kernel (1) is even (or odd), that is, if $\Omega(-x)=\Omega(x)$, $x \in \mathbf{R}^{n} \backslash\{0\}$ (or $\Omega(-x)=-\Omega(x), x \in \mathbf{R}^{n} \backslash\{0\}$ ). Let $T^{*}$ be the maximal singular integral

$$
T^{*} f(x)=\sup _{\varepsilon>0}\left|T^{\varepsilon} f(x)\right|, \quad x \in \mathbf{R}^{n} .
$$

The classical Cotlar's inequality (e.g. [Gr1, p. 295]) asserts that

$$
T^{*} f(x) \leq C(M(T f)(x)+M(f)(x)), \quad x \in \mathbf{R}^{n},
$$

where $M$ denotes the Hardy-Littlewood maximal operator. In [MV] one proved the pointwise inequality

$$
B^{*} f(z) \leq M(B f)(z), \quad z \in \mathbf{C},
$$

where $B f$ is the Beurling transform of $f$ defined by

$$
B f(z)=\text { p.v. } \frac{-1}{\pi} \int \frac{f(z-w)}{w^{2}} d w, \quad z \in \mathbf{C},
$$

$d w$ denoting the 2-dimensional Lebesgue measure. The work [MOV] addresses the issue of characterizing even smooth homogeneous Calderón-Zygmund operators for which the inequality (4) is true. In particular, it shows that $T^{*} f(x) \leq C M(T f)(x)$, $x \in \mathbf{R}^{n}$, if and only if $\left\|T^{*} f\right\|_{2} \leq C\|T f\|_{2}, f \in L^{2}\left(\mathbf{R}^{n}\right)$. The analogous question for odd operators is treated in [MOPV], showing that $T^{*} f(x) \leq C M^{2}(T f)(x), x \in \mathbf{R}^{n}$, if and only if $\left\|T^{*} f\right\|_{2} \leq C\|T f\|_{2}, f \in L^{2}\left(\mathbf{R}^{n}\right)$. (See [BMO] for an $L^{p}$ version of these results.)

In the definition (3) of the truncated operator $T^{\varepsilon} f$, the kernel $K$ is supported outside a ball of radius $\varepsilon$. It is natural to consider the truncation outside a cube of side length $\varepsilon$, that is, let $Q(x, \varepsilon)$ be the cube, with sides parallel to axis, centered at $x$ of side length $\varepsilon$ and define

$$
T_{Q}^{\varepsilon} f(x)=\int_{y \notin Q(0, \varepsilon)} f(x-y) K(y) d y=\int_{y \notin Q(x, \varepsilon)} f(y) K(x-y) d y,
$$

and consider the associated maximal singular integral

$$
T_{S}^{*} f(x)=\sup _{\varepsilon>0}\left|T_{Q}^{\varepsilon} f(x)\right|, \quad x \in \mathbf{R}^{n} .
$$

By simple geometry one checks that

$$
\begin{aligned}
& T_{Q}^{\varepsilon} f(x)=T^{\sqrt{n} \varepsilon / 2} f(x)+\int_{B(0, \sqrt{n} \varepsilon / 2) \backslash Q(0, \varepsilon)} f(x-y) K(y) d y, \\
& T^{\varepsilon} f(x)=T_{Q}^{2 \varepsilon} f(x)+\int_{Q(0,2 \varepsilon) \backslash B(0, \varepsilon)} f(x-y) K(y) d y,
\end{aligned}
$$

and therefore

$$
\begin{aligned}
& T_{S}^{*} f(x) \leq T^{*} f(x)+C M(f)(x), \\
& T^{*} f(x) \leq T_{S}^{*} f(x)+C M(f)(x) .
\end{aligned}
$$

Consequently, from the $L^{p}$-theory point of view, the maximal operators $T^{*}$ and $T_{S}^{*}$ are equivalent. The problem we address is the study of pointwise inequalities that relate $T_{S}^{*} f(x)$ with $T f(x)$ like in (4). In this note we provide an answer for the Beurling transform defined in (5) and for its $k$ th iteration $B^{k}=B \circ \cdots \circ B$. Before estating our results, let us recall a few facts concerning the Beurling transform. The 
Fourier multiplier of $B$ is $\frac{\bar{\xi}}{\xi}$, that is, $\widehat{B f}(\xi)=\frac{\bar{\xi}}{\xi} \hat{f}(\xi)$, and then $B$ is an isometry on $L^{2}(\mathbf{C})$. The kernel $b_{k}$ of $B^{k}$ can be computed explicitly, for instance via a Fourier transform argument [St, p. 73], and one obtains

$$
b_{k}(z)=\frac{(-1)^{k} k}{\pi} \frac{\bar{z}^{k-1}}{z^{k+1}}, \quad z \neq 0 .
$$

Similarly we get that the kernel of the inverse operator, $\left(B^{k}\right)^{-1}$, is precisely the conjugate kernel: $\frac{(-1)^{k} k}{\pi} \frac{z^{k-1}}{\bar{z}^{k+1}}$.

Theorem. (a) If $k$ is odd, then

$$
\left(B^{k}\right)_{S}^{*} f(z) \leq C M^{2}\left(B^{k} f\right)(z), \quad z \in \mathbf{C},
$$

where $C$ is a constant depending on $k$ and $M^{2}=M \circ M$ is the iterated maximal operator.

(b) If $k$ is even, for any positive constant $C$, for any $z \in \mathbf{C}$ and for any positive integer $j$ there exists a function $f$ in $L^{2}(\mathbf{C})$ such that

$$
\left(B^{k}\right)_{S}^{*} f(z) \geq C M^{j}\left(B^{k} f\right)(z),
$$

where $M^{j}=M \circ \cdots \circ M$ is the $j$ th iteration of $M$.

In the right hand side of (4) there is the maximal operator, but in (a) of the Theorem the iteration of $M$ appears. The reason is that, for $k$ odd, $\left(B^{k}\right)^{-1}\left(b_{k} \chi_{\mathbf{C} \backslash B(0,1)}\right)$ is a bounded function with compact support, while $\left(B^{k}\right)^{-1}\left(b_{k} \chi_{\mathbf{C} \backslash Q(0,1)}\right)$ is an unbounded BMO function with unbounded support. We also provide an example to show that in part (a) of the above Theorem for $k=1$ we cannot replace the iterated Hardy-Littlewood maximal operator $M^{2}=M \circ M$ by $M$. We believe that similar examples should also give optimality for the case $k=3,5, \ldots$. Property (b) of the Theorem also holds for the Cauchy transform on a Lipschitz graph when the graph is not a line (see $[\mathrm{Gi}]$ ).

We adhere to the usual convention of denoting by $C$ a positive constant, independent of the relevant parameters involved, and which may vary from an occurrence to another. Our notation and terminology are standard. For example, $A \approx B$ means that the two quantities $A$ and $B$ satisfy the relation $C^{-1} A \leq B \leq C A$, for some constant $C \geq 1$.

\section{Proof of the Theorem}

We proceed as in $[\mathrm{MOPV}]$. We start without assuming any parity on $k$. By translating and dilating one reduces the proof to

$$
\left|\left(B^{k}\right)_{Q}^{2} f(0)\right| \leq C M^{2}\left(B^{k} f\right)(0),
$$

where $\left(B^{k}\right)_{Q}^{2} f(0)$ is the truncated integral at level 2. Denote the square $Q(0,2)=$ $[-1,1] \times[-1,1]$ by $Q_{0}$. As usual, $\chi_{E}$ denotes the characteristic function of the set $E$. The idea is to obtain an identity of the form

$$
b_{k}(z) \chi_{\mathbf{C} \backslash Q_{0}}(z)=B^{k}\left(a_{k}\right)(z),
$$

for some function $a_{k}$. Since $B^{k}$ is an invertible operator, we have

$$
a_{k}=\left(B^{k}\right)^{-1}\left(b_{k} \chi_{\mathbf{C} \backslash Q_{0}}\right)
$$


and so $a_{k} \in \operatorname{BMO}(\mathbf{C})$. We claim that if $k$ is odd we have the decay estimate

$$
\left|a_{k}(z)\right| \leq \frac{C_{k}}{|z|^{3}}, \quad \text { if }|z|>3
$$

Before proving the claim we show how it yields (a) of the Theorem. We argue as in [MOPV, p. 3675]. For any $f \in L^{2}(\mathbf{C})$ we have

$$
\begin{aligned}
\left(B^{k}\right)_{Q}^{2} f(0)= & \int_{z \notin Q_{0}} f(z) b_{k}(-z) d z=\int f(z) b_{k}(z) \chi_{\mathbf{C} \backslash Q_{0}}(z) d z \\
= & \int f(z) B^{k}\left(a_{k}\right)(z) d z=\int B^{k} f(z) a_{k}(z) d z \\
= & \int_{|z|<3} B^{k} f(z)\left(a_{k}(z)-\left(a_{k}\right)_{B(0,3)}\right) d z \\
& +\left(a_{k}\right)_{B(0,3)} \int_{|z|<3} B^{k} f(z) d z+\int_{|z|>3} B^{k} f(z) a_{k}(z) d z \\
:= & I+I I+I I I,
\end{aligned}
$$

where, as usual, $\left(a_{k}\right)_{B(0,3)}=|B(0,3)|^{-1} \int_{B(0,3)} a_{k}(z) d z$. To estimate the term $I$ we use the generalized Hölder's inequality and the pointwise equivalence $M_{L(\log L)} f(x) \approx$ $M^{2} f(x)([\mathrm{P}])$ to get

$$
|I| \leq C\left\|a_{k}\right\|_{\mathrm{BMO}}\left\|B^{k} f\right\|_{L(\log L), B(0,3)} \leq C M^{2}\left(B^{k} f\right)(0) .
$$

Clearly,

$$
|I I| \leq C M\left(B^{k} f\right)(0) .
$$

Finally, from the decay of $a_{k}$ we obtain

$$
|I I I| \leq C \int_{|z|>3} \frac{\left|B^{k} f(z)\right|}{|z|^{3}} d y \leq C M\left(B^{k} f\right)(0),
$$

by using a standard argument which consists in estimating the integral on the annuli $\left\{3^{j} \leq|z|<3^{j+1}\right\}$. Therefore we get (6) and part (a) of the Theorem is proved. We turn now to the proof of the claim. We express $a_{k}$ as

$$
a_{k}=\left(B^{k}\right)^{-1}\left(b_{k} \chi_{\mathbf{C} \backslash Q_{0}}\right)=\left(B^{k}\right)^{-1}\left(b_{k}-b_{k} \chi_{Q_{0}}\right)=\delta_{0}-\left(B^{k}\right)^{-1}\left(b_{k} \chi_{Q_{0}}\right),
$$

where $\delta_{0}$ is the Dirac delta at the origin. We have to determine the decay of $\left(B^{k}\right)^{-1}\left(b_{k} \chi_{Q_{0}}\right)(z)$ when $|z|>3$ :

$$
\begin{aligned}
\left(B^{k}\right)^{-1}\left(b_{k} \chi_{Q_{0}}\right)(z)= & \lim _{\varepsilon \rightarrow 0} \int_{\varepsilon<|w|} \overline{b_{k}(z-w)}\left(b_{k} \chi_{Q_{0}}\right)(w) d w \\
= & \lim _{\varepsilon \rightarrow 0} \int_{\varepsilon<|w|}\left(\overline{b_{k}(z-w)}-\overline{b_{k}(z)}\right)\left(b_{k} \chi_{Q_{0}}\right)(w) d w \\
& \quad+\overline{b_{k}(z)} \lim _{\varepsilon \rightarrow 0} \int_{\varepsilon<|w|}\left(b_{k} \chi_{Q_{0}}\right)(w) d w \\
= & \lim _{\varepsilon \rightarrow 0} \int_{\varepsilon<|w|}\left(\overline{b_{k}(z-w)}-\overline{b_{k}(z)}\right)\left(b_{k} \chi_{Q_{0}}\right)(w) d w+\overline{b_{k}(z)} B^{k}\left(\chi_{Q_{0}}\right)(0) \\
:= & I_{k}+I I_{k} .
\end{aligned}
$$


On the one hand, since

$$
\left|\overline{b_{k}(z-w)}-\overline{b_{k}(z)}\right| \leq \frac{C|w|}{|z|^{3}}, \quad|z|>3, \quad|w| \leq \sqrt{2}
$$

we obtain

$$
\left|I_{k}\right| \leq C \int_{w \in Q_{0}} \frac{|w|}{|z|^{3}} \frac{1}{|w|^{2}} d w=\frac{C}{|z|^{3}}
$$

On the other hand, we will check in the next Lemma that when $k$ is odd $B^{k}\left(\chi_{Q_{0}}\right)(0)=$ 0 . Then $I I_{k}=0$ and we get $(7)$.

Lemma. Let $Q$ be any square, with sides parallel to axis, centered at 0 . Then

(a) $\overline{\left(B^{k} \chi_{Q}\right)(z)}=\left(B^{k} \chi_{Q}\right)(\bar{z})$.

(b) $\left(B^{k} \chi_{Q}\right)(i z)=(-1)^{k}\left(B^{k} \chi_{Q}\right)(z)$.

(c) $\left(B^{k} \chi_{Q}\right)(0)=0$ if $k$ is odd, and $\left(B^{k} \chi_{Q}\right)(0) \neq 0$ if $k$ is even.

Proof. It is easy to check (a) and (b). Just consider the symmetry of the domain with respect to conjugation and with respect to a rotation of angle $\pi / 2$ :

$$
\begin{aligned}
\overline{\left(B^{k} \chi_{Q}\right)(z)} & =\text { p.v. } \int_{Q} \overline{b_{k}(z-w)} d w=\text { p.v. } \int_{Q} b_{k}(\bar{z}-\bar{w}) d w \\
& =\text { p.v. } \int_{Q} b_{k}(\bar{z}-w) d w=\left(B^{k} \chi_{Q}\right)(\bar{z})
\end{aligned}
$$

and

$$
\begin{aligned}
\left(B^{k} \chi_{Q}\right)(i z) & =\text { p.v. } \int_{Q} b_{k}(i z-w) d w=\text { p.v. } \int_{Q} b_{k}(i z-i w) d w \\
& =(-i)^{2 k} \text { p.v. } \int_{Q} b_{k}(z-w) d w=(-1)^{k}\left(B^{k} \chi_{Q}\right)(z) .
\end{aligned}
$$

From (b), when $k$ is odd, we have $\left(B^{k} \chi_{Q}\right)(0)=-\left(B^{k} \chi_{Q}\right)(0)$ and so $\left(B^{k} \chi_{Q}\right)(0)=0$. To get $\left(B^{k} \chi_{Q}\right)(0) \neq 0$ when $k$ is even we have to work a little bit more. Set $k=2 j$ and by simplicity let $Q$ be the square $Q_{0}$. Then

$$
\begin{aligned}
\left(B^{2 j} \chi_{Q_{0}}\right)(0) & =\text { p.v. } \int_{Q_{0}} b_{2 j}(w) d w=\int_{Q_{0} \backslash B(0,1)} b_{2 j}(w) d w \\
& =4 \int_{E} b_{2 j}(w) d w=4 \frac{2 j}{\pi} \int_{E} \frac{\bar{w}^{2 j-1}}{w^{2 j+1}} d w
\end{aligned}
$$

where $E=\left\{w \in Q_{0} \backslash B(0,1): \operatorname{Re} w>0\right.$ and $\left.\operatorname{Im} w>0\right\}$. The second identity follows from the cancellation of the kernels and the third one from $b_{2 j}(w)=b_{2 j}(i w)$. Therefore, making the change to polar coordinates $w=r e^{i \theta}$, with $1 \leq r \leq \sqrt{2}$ and $\theta(r) \leq \theta \leq \frac{\pi}{2}-\theta(r), \theta(r)=\arccos \left(\frac{1}{r}\right)$, we have

$$
\begin{aligned}
\left(B^{2 j} \chi_{Q_{0}}\right)(0) & =4 \frac{2 j}{\pi} \int_{1}^{\sqrt{2}} \int_{\theta(r)}^{\frac{\pi}{2}-\theta(r)} \frac{\left(r e^{-i \theta}\right)^{2 j-1}}{\left(r e^{i \theta}\right)^{2 j+1}} d \theta r d r \\
& =4 \frac{2 j}{\pi} \int_{1}^{\sqrt{2}} \int_{\theta(r)}^{\frac{\pi}{2}-\theta(r)} e^{-i \theta 4 j} d \theta \frac{d r}{r}=\frac{-4}{\pi} \int_{1}^{\sqrt{2}} \sin (\theta(r) 4 j) \frac{d r}{r} .
\end{aligned}
$$


Expressing $\sin (\theta(r) 4 j)$ in terms of $\sin (\theta(r))$ and $\cos (\theta(r))$ and replacing $\sin (\theta(r))=$ $\frac{\sqrt{r^{2}-1}}{r}$ and $\cos (\theta(r))=1 / r$ we get

$$
\begin{aligned}
\left(B^{2 j} \chi_{Q_{0}}\right)(0) & =\frac{-4}{\pi} \sum_{m=0}^{2 j-1}(-1)^{m}\left(\begin{array}{c}
4 j \\
2 m+1
\end{array}\right) \int_{1}^{\sqrt{2}} \frac{1}{r^{4 j}}\left(r^{2}-1\right)^{\frac{2 m+1}{2}} \frac{d r}{r} \\
& =\frac{-4}{\pi} \sum_{m=0}^{2 j-1}(-1)^{m}\left(\begin{array}{c}
4 j \\
2 m+1
\end{array}\right) \int_{0}^{1} \frac{x^{2 m+2}}{\left(x^{2}+1\right)^{2 j+1}} d x \\
& :=\frac{-4}{\pi} \int_{0}^{1} F_{j}(x) d x .
\end{aligned}
$$

Integrating by parts as many times as necessary, one easily obtains, for some $a, b \in \mathbf{Q}$,

$$
\int_{0}^{1} \frac{x^{2 m+2}}{\left(x^{2}+1\right)^{2 j+1}} d x=a+b \frac{\pi}{4} \neq 0 .
$$

Although each factor in (10) is non-zero, there still might be a cancellation in the sum on $m$. We will check that $\left(B^{2 j} \chi_{Q_{0}}\right)(0) \neq 0$ by proving that a primitive of $F_{j}(x)$ is some rational function $R_{j}(x)$, with integer coefficients, minus $\arctan (x)$. Thus we have

$$
\int_{0}^{1} F_{j}(x) d x=R_{j}(1)-R_{j}(0)-\frac{\pi}{4} \neq 0,
$$

because $R_{j}(1)-R_{j}(0)$ is a rational number.

Given $n<d$, we define

$$
I(d, 2 n):=\int \frac{x^{2 n}}{\left(x^{2}+1\right)^{d}} d x .
$$

It is not necessary to fix the constant of the primitive because we will value a definite integral. Integrating by parts we have

$$
I(d, 2 n)=\frac{-x^{2 n-1}}{2(d-1)\left(x^{2}+1\right)^{d-1}}+\frac{2 n-1}{2(d-1)} I(d-1,2(n-1)) .
$$

Iterating the procedure we obtain

$$
I(d, 2 n)=\frac{(2 n-1) !}{2^{n-1}(n-1) !} \frac{(d-n-1) !}{2^{n}(d-1) !} I(d-n, 0)+R(x)
$$

where $R(x)$ is some rational function defined on $\mathbf{R}$ and $R(0)=0$. Given $k \geq 2$ one easily gets

$$
I(k, 0)=\frac{(2 k-3) !}{2^{2 k-3}(k-1) !(k-2) !} I(1,0)+R(x) .
$$

Now, from (10) together with (11) and (12),

$$
\begin{aligned}
\int F_{j}(x) d x=I(1,0)\{ & \left(\sum_{m=0}^{2 j-2}(-1)^{m}\left(\begin{array}{c}
4 j \\
2 m+1
\end{array}\right) \frac{(2 m+1) !(4 j-2 m-3) !}{m !(2 j) !(2 j-m-2) ! 2^{4 j-2}}\right) \\
& \left.-\frac{(4 j) !}{(2 j-1) !(2 j) ! 2^{4 j-1}}\right\}+R_{j}(x) .
\end{aligned}
$$


Performing some computations (see the Appendix), we get exactly what we wanted,

$$
\left(\sum_{m=0}^{2 j-2}(-1)^{m}\left(\begin{array}{c}
4 j \\
2 m+1
\end{array}\right) \frac{(2 m+1) !(4 j-2 m-3) !}{m !(2 j) !(2 j-m-2) ! 2^{4 j-2}}\right)-\frac{(4 j) !}{(2 j-1) !(2 j) ! 2^{4 j-1}}=-1
$$

that is,

$$
\int F_{j}(x) d x=-I(1,0)+R_{j}(x)=-\arctan (x)+R_{j}(x) .
$$

Let us prove assertion (b) of the Theorem. Recall that now $k$ is even. By (8) one has

$$
\left|\left(B^{k}\right)_{Q}^{2} f(0)\right| \lesssim M^{j}\left(B^{k} f\right)(0)
$$

if and only if

$$
|I I I| \lesssim M^{j}\left(B^{k} f\right)(0)
$$

By (9), when $|z|>3$,

$$
a_{k}(z)=\overline{b_{k}(z)}\left(B^{k} \chi_{Q_{0}}\right)(0)+O\left(\frac{1}{|z|^{3}}\right):=\alpha_{k} \frac{z^{k-1}}{\bar{z}^{k+1}}+O\left(\frac{1}{|z|^{3}}\right),
$$

where $\alpha_{k}$ is a non-zero constant that depends on $k$. Consequently, (14) holds if and only if

$$
\left|\int_{|z|>3} \frac{z^{k-1}}{\bar{z}^{k+1}} B^{k} f(z) d z\right| \lesssim M^{j}\left(B^{k} f\right)(0), \quad f \in L^{2} .
$$

Since that $B^{k}$ is invertible in $L^{2}$, this is equivalent to

$$
\left|\int_{|z|>3} \frac{z^{k-1}}{\bar{z}^{k+1}} G(z) d z\right| \lesssim M^{j}(G)(0), \quad G \in L^{2} .
$$

But (15) is false. Indeed, let $G$ be a function with compact support and $0 \leq G \leq 1$. Obviously, $M^{j}(G) \leq 1$. On the other hand, since $\frac{z^{k-1}}{\bar{z}^{k+1}} \chi_{B(0,3)^{c}}(x)$ does not belong to $L^{1}(\mathbf{C})$, we can make the left-hand side of $(15)$ as big as we want.

\section{Counterexample}

This section is devoted to prove that condition (a) in the Theorem is sharp for $k=1$. More precisely, we will prove that there exists a function $f$ such that for each constant $C>0$ there exists a point $z \in \mathbf{C}$ satisfying

$$
B_{S}^{*} f(z)>C M(B f)(z) \text {. }
$$

We choose $f:=B^{-1}\left(\chi_{Q_{0}}\right)$. For $|z|>2$ one has $M(B f)(z)=M\left(\chi_{Q_{0}}\right)(z) \approx \frac{1}{|z|^{2}}$. So, in order to get inequality (16) it is sufficient to prove that, for some $z$, one has

$$
B_{S}^{*} f(z) \geq C \frac{\log |z|}{|z|^{2}} .
$$

For $m>2$ (for instance, $m=5$ ), take $\alpha \gg m$, set $z=\alpha+i \alpha$ and consider the truncated operator

$$
(B)_{Q}^{2(\alpha+m)} f(z)=\frac{-1}{\pi} \int_{w \notin Q(z, 2(\alpha+m))} \frac{f(w)}{(z-w)^{2}} d w .
$$


By definition $B_{Q}^{*} f(z) \geq\left|(B)_{Q}^{2(\alpha+m)} f(z)\right|$ and then we will have (17) if we prove

$$
\left|(B)_{Q}^{2(\alpha+m)} f(z)\right| \gtrsim \frac{\log |z|}{|z|^{2}} .
$$

The idea is to decompose $(B)_{Q}^{2(\alpha+m)} f(z)$ as a sum of certain terms. All terms, except one, can be bounded by $C|z|^{-2}$ and the exceptional term will be of order $|z|^{-2} \log |z|$. We begin by writing the equality

$$
(B)_{Q}^{2(\alpha+m)} f(z)=B^{\sqrt{2}(\alpha+m)} f(z)-\frac{1}{\pi} \int_{E} \frac{f(w)}{(w-z)^{2}} d w
$$

where $E$ is the set $B(z, \sqrt{2}(\alpha+m)) \backslash Q(z, 2(\alpha+m))$. From the pointwise inequality (4) the first term is bounded by $\frac{C}{|z|^{2}}$ and we just care about the second one. Set $E=$ $A_{1} \cup A_{2} \cup B$ where (see Figure 1)

$$
\begin{aligned}
A_{1} & :=\{w \in E: \operatorname{Re}(w-z)<0, \operatorname{Im}(w-z)<0 \text { and } \operatorname{Im}(w+m+i m)>0\}, \\
A_{2} & :=\{w \in E: \operatorname{Re}(w-z)<0, \operatorname{Im}(w-z)<0 \text { and } \operatorname{Im}(w+m+i m)<0\}, \\
B & :=E \backslash\left(A_{1} \cup A_{2}\right) .
\end{aligned}
$$

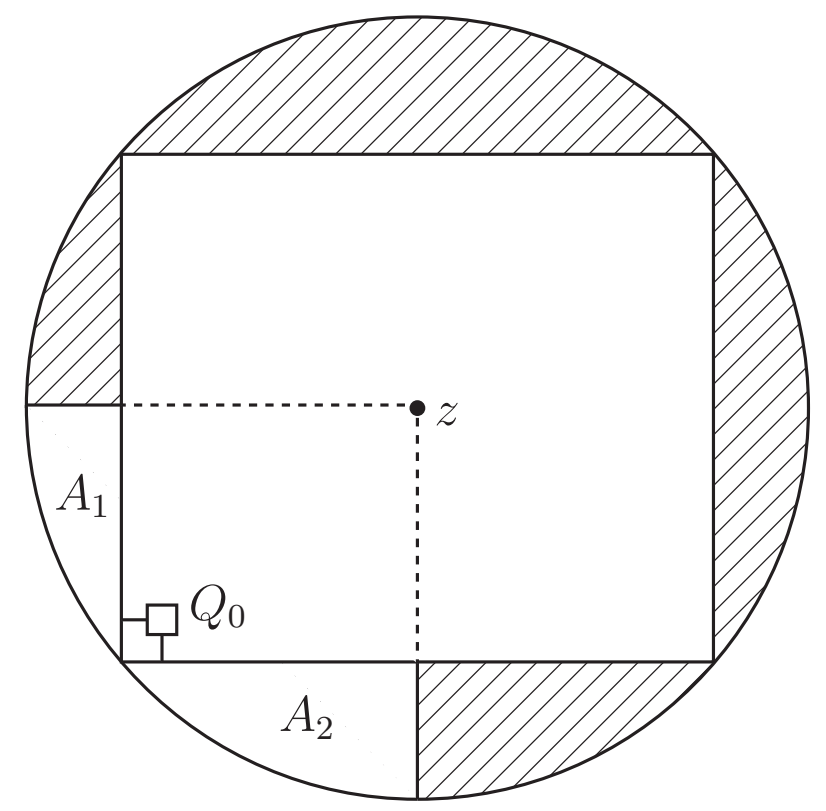

Figure 1.

Thus

$$
\int_{E} \frac{f(w)}{(w-z)^{2}} d w=\int_{A_{1} \cup A_{2}} \frac{f(w)}{(w-z)^{2}} d w+\int_{B} \frac{f(w)}{(\xi-z)^{2}} d w .
$$

On the set $B$ we have $|f(w)|=\left|B^{-1}\left(\chi_{Q_{0}}\right)(w)\right| \leq \frac{C}{|w|^{2}}$ and then

$$
\left|\int_{B} \frac{f(w)}{(w-z)^{2}} d w\right| \leq \int_{B} \frac{C}{|w-z|^{2}|w|^{2}} d w \leq \frac{C|B|}{|z|^{4}}=\frac{C}{|z|^{2}}
$$

So, to get (18) it remains to prove that

$$
\left|\int_{A_{1} \cup A_{2}} \frac{f(w)}{(w-z)^{2}} d w\right| \geq C \frac{\log |z|}{|z|^{2}} .
$$


For any $w \in A_{1} \cup A_{2}$ we write

$$
f(w)=\frac{-1}{\pi} \int_{Q_{0}} \frac{1}{(\overline{w-\xi})^{2}} d \xi=\frac{-1}{\pi} \frac{\left|Q_{o}\right|}{\bar{w}^{2}}-\frac{1}{\pi} \int_{Q_{0}}\left(\frac{1}{(\overline{w-\xi})^{2}}-\frac{1}{\bar{w}^{2}}\right) d \xi .
$$

By the mean value property, the last integral in the above equality is bounded by $\frac{C}{|w|^{3}}$. Therefore, an easy computation gives

$$
\left|\int_{A_{1} \cup A_{2}}\left(f(w)+\frac{1}{\pi} \frac{\left|Q_{0}\right|}{\bar{w}^{2}}\right) \frac{1}{(w-z)^{2}} d w\right| \leq C \int_{A_{1} \cup A_{2}} \frac{1}{|w|^{3}} \frac{1}{|w-z|^{2}} d w \leq \frac{C}{|z|^{2}} .
$$

Consequently, inequality (19) holds if and only if

$$
\left|\int_{A_{1} \cup A_{2}} \frac{1}{(z-w)^{2}} \frac{1}{\bar{w}^{2}} d w\right| \geq C \frac{\log |z|}{|z|^{2}} .
$$

Notice that, by the symmetry of the sets, $w \in A_{1}$ if and only if $i \bar{w} \in A_{2}$. Thus the integral in (20) can be written as

$$
\int_{A_{1}}\left(\frac{1}{(z-w)^{2}} \frac{1}{\bar{w}^{2}}+\frac{1}{(z-i \bar{w})^{2}} \frac{1}{(i w)^{2}}\right) d w
$$

Set $A_{1}^{-}=\left\{w \in A_{1}: \operatorname{Im}(w)<0\right\}, A_{1}^{+}=\left\{w \in A_{1}: \bar{w} \in A_{1}^{-}\right\}$and let $D=$ $A_{1} \backslash\left(A_{1}^{+} \cup A_{1}^{-}\right)$(see Figure 2).

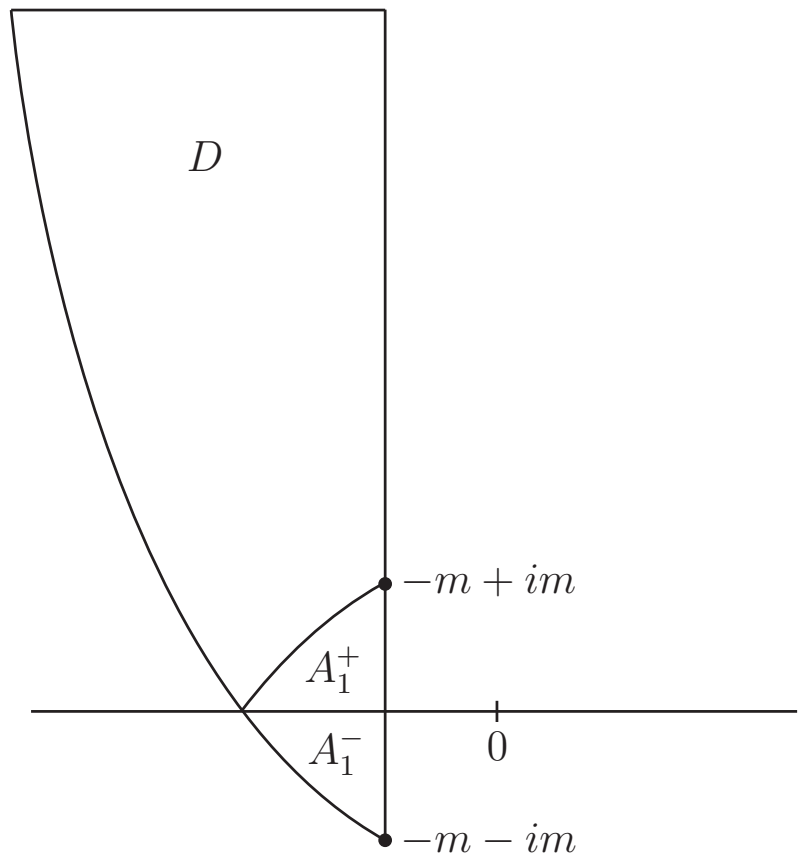

Figure 2.

First of all we prove that the integral over $A_{1}^{+} \cup A_{1}^{-}$is bounded by $\frac{C}{|z|^{2}}$. Using a change of variable in the integral over $A_{1}^{-}$, one can see that the integral over $A_{1}^{+} \cup A_{1}^{-}$ is

$$
\int_{A_{1}^{+}}\left(\frac{1}{(z-\bar{w})^{2}}-\frac{1}{(z-i \bar{w})^{2}}\right) \frac{1}{w^{2}} d w+\int_{A_{1}^{+}}\left(\frac{1}{(z-w)^{2}}-\frac{1}{(z-i w)^{2}}\right) \frac{1}{\bar{w}^{2}} d w:=I+I I .
$$


We are going to estimate $|I|$ and $|I I|$ by $\frac{C}{|z|^{2}}$. Indeed,

$$
|I|=\left|\int_{A_{1}^{+}} \frac{-2 \bar{w}^{2}+\bar{w}(2 z-2 i z)}{w^{2}(z-\bar{w})^{2}(z-i \bar{w})^{2}} d w\right| \leq \frac{C}{|z|^{4}} \int_{A_{1}^{+}}\left(1+\frac{|z|}{|w|}\right) d w \leq \frac{C m}{|z|^{3}} \leq \frac{C}{|z|^{2}}
$$

where in the first inequality we have used that $|z-\bar{w}|=|z-i \bar{w}| \approx|z|$, and in the second one that $\int_{A_{1}^{+}}|w|^{-1} \leq C m$. Using similar computations for the second term we get

$$
|I I|=\left|\int_{A_{1}^{+}} \frac{-2 w^{2}+w(2 z-2 i z)}{\bar{w}^{2}(z-w)^{2}(z-i w)^{2}} d w\right| \leq \frac{C}{|z|^{2}}
$$

So, only remains to compute the integral over $D$, which we split again into two terms,

$$
\begin{aligned}
& \int_{D}\left(\frac{1}{(z-w)^{2}} \frac{1}{\bar{w}^{2}}-\frac{1}{(z-i \bar{w})^{2}} \frac{1}{w^{2}}\right) d w=\int_{D} \frac{1}{(z-w)^{2}}\left(\frac{1}{\bar{w}^{2}}-\frac{1}{w^{2}}\right) d w \\
& +\int_{D} \frac{1}{w^{2}}\left(\frac{1}{(z-w)^{2}}-\frac{1}{(z-i \bar{w})^{2}}\right) d w:=I I I+I V .
\end{aligned}
$$

We note that if $w \in D$ then $|z-\bar{w}|=|z-i \bar{w}| \approx|z|$ and $m \leq|w| \leq 3|z|$. We treat the term $|I V|$ as before. In fact, we have

$$
\begin{aligned}
|I V| & =\int_{D} \frac{2 z(w-i \bar{w})-\bar{w}^{2}-w^{2}}{w^{2}(z-w)^{2}(z-i \bar{w})^{2}} d w \leq \int_{D} \frac{2|w|^{2}+4|z||w|}{|w|^{2}|z-w|^{2}|z-i \bar{w}|^{2}} d w \\
& \leq \frac{C}{|z|^{4}} \int_{D}\left(1+\frac{|z|}{|w|}\right) d w \leq \frac{C|D|}{|z|^{4}}+\frac{C}{|z|^{3}} \int_{m}^{3|z|} 1 d r \leq \frac{C}{|z|^{2}} .
\end{aligned}
$$

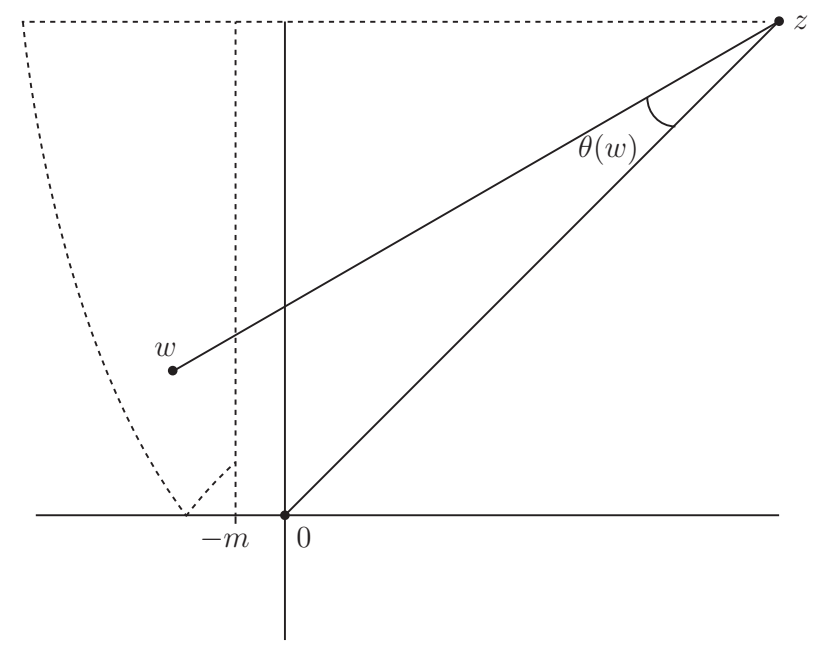

Figure 3 .

Finally the term $I I I$ will give us that our counterexample works. For $w \in D$ (see Figure 3) we write $w-z=R(w) e^{i\left(\frac{5 \pi}{4}-\theta(w)\right)}$ where $R(w)=|w-z| \approx|z|$ and 
$0 \leq \theta(w) \leq \frac{\pi}{4}$. So, $i(w-z)^{-2}=-e^{i 2 \theta(w)}(R(w))^{-2}$. Then we have

$$
\begin{aligned}
I I I & =2 i \int_{D} \frac{1}{(z-w)^{2}} \frac{\operatorname{Im} w^{2}}{|w|^{4}} d w=-2 \int_{D} \frac{e^{i 2 \theta(w)}}{R^{2}(w)} \frac{\operatorname{Im} w^{2}}{|w|^{4}} d w \\
& =-2 \int_{D} \frac{(\cos 2 \theta(w)+i \sin 2 \theta(w))}{R^{2}(w)} \frac{\operatorname{Im} w^{2}}{|w|^{4}} d w .
\end{aligned}
$$

Since $\cos 2 \theta(w) \geq 0$ and $\operatorname{Im} w^{2} \leq 0$,

$$
|\operatorname{Re} I I I|=2 \int_{D} \frac{\cos 2 \theta(w)}{R^{2}(w)} \frac{\left|\operatorname{Im} w^{2}\right|}{|w|^{4}} d w \geq \frac{C}{|z|^{2}} \int_{D} \cos 2 \theta(w) \frac{\left|\operatorname{Im} w^{2}\right|}{|w|^{4}} d w .
$$

Fix $\delta>0$ and set

$$
\widetilde{D}:=\{w \in D: \cos 2 \theta(w)>\delta\} \cap\left\{w \in \mathbf{C}: \frac{\pi}{2}+\frac{\pi}{100} \leq \arg w \leq \pi-\frac{\pi}{100}\right\} .
$$

Then, if $w \in \widetilde{D}$ we have

$$
|\operatorname{Im} w| \geq|w| \frac{\beta}{\sqrt{1+\beta^{2}}} \geq \frac{\beta|w|}{2}
$$

where $\beta=\tan \frac{\pi}{50}$. Note that the Lebesgue measure of $\widetilde{D}$ is comparable to the Lebesgue measure of $D$. Finally, using polar coordinates we get

$$
|\operatorname{Re} I I I| \geq C \frac{\delta \beta}{|z|^{2}} \int_{\widetilde{D}} \frac{1}{|w|^{2}} d w \geq C \frac{\delta \beta}{|z|^{2}} \int_{4 m}^{|z| / 10} \frac{d r}{r} \geq C \frac{\delta \beta}{|z|^{2}} \log |z|,
$$

which yields (20) and the counterexample.

\section{Appendix}

In this section we will prove, for the sake of the reader's convenience, identity (13). To get it we will write the term in the left hand side of (13) in another way. That is,

$$
\begin{gathered}
\left(\sum_{m=0}^{2 j-2}(-1)^{m}\left(\begin{array}{c}
4 j \\
2 m+1
\end{array}\right) \frac{(2 m+1) !(4 j-2 m-3) !}{m !(2 j) !(2 j-m-2) ! 2^{4 j-2}}\right)-\frac{(4 j) !}{(2 j-1) !(2 j) ! 2^{4 j-1}} \\
=\frac{(4 j) !}{(2 j) !(2 j-1) ! 2^{4 j-1}} \sum_{m=0}^{2 j-1}(-1)^{m}\left(\begin{array}{c}
2 j-1 \\
m
\end{array}\right) \frac{1}{4 j-2 m-1} \\
:=\frac{(4 j) !}{(2 j) !(2 j-1) ! 2^{4 j-1}} S,
\end{gathered}
$$

where the last identity defines $S$. Thus, only remains to prove that

$$
S=-\frac{(4 j) !}{(2 j) !(2 j-1) ! 2^{4 j-1}} \text {. }
$$

Using twice the trivial fact that

$$
\sum_{m=0}^{2 j-1}(-1)^{m}\left(\begin{array}{c}
2 j-1 \\
m
\end{array}\right)=(1-1)^{2 j-1}=0,
$$


we have

$$
\begin{aligned}
S & =\sum_{m=0}^{2 j-1}(-1)^{m}\left(\begin{array}{c}
2 j-1 \\
m
\end{array}\right)\left(\frac{1}{4 j-2 m-1}-1\right) \\
& =(-1) 2(2 j-1) \sum_{m=0}^{2 j-2}(-1)^{m}\left(\begin{array}{c}
2 j-1 \\
m
\end{array}\right) \frac{1}{4 j-2 m-1} \\
& =(-1) 2(2 j-1) \sum_{m=0}^{2 j-2}(-1)^{m}\left(\begin{array}{c}
2 j-1 \\
m
\end{array}\right)\left(\frac{1}{4 j-2 m-1}-\frac{1}{3}\right) \\
& =(-1)^{2} 2^{2}(2 j-1)(2 j-2) \sum_{m=0}^{2 j-3}(-1)^{m}\left(\begin{array}{c}
2 j-1 \\
m
\end{array}\right) \frac{1}{4 j-2 m-1} .
\end{aligned}
$$

Iterating this process $(2 j-1)$ times we obtain $(21)$, and so (13) is proved.

Acknowledgements. The authors were partially supported by grants numbers 2014 SGR 75 (AGAUR) and MTM2013-44699-P (MINECO). The first author thanks the support received by Ferran Sunyer i Balaguer Foundation in the preparation of this work.

\section{References}

[BMO] Bosch-Camós, A., J. Mateu, and J. Orobitg: $L^{p}$ estimates for the maximal singular integral in terms of the singular integral. - J. Anal. Math. (to appear).

[Gi] Girela-Sarrión, D.: Counterexamples to some pointwise estimates of the maximal Cauchy transform in terms of the Cauchy transform. - Ann. Acad. Sci. Fenn. Math. 38:2, 2013, 657-675.

[Gr1] Grafakos, L.: Classical Fourier analysis. - Grad. Texts in Math. 249, Springer Verlag, Berlin, Second Edition, 2008.

[Mopv] Mateu, J., J. Orobitg, C. Perez, and J. Verdera: New estimates for the maximal singular integral. - Int. Math. Res. Not. IMRN 19, 2010, 3658-3722.

[MOV] Mateu, J., J. Orobitg, and J. Verdera: Estimates for the maximal singular integral in terms of the singular integral: the case of even kernels. - Ann. of Math. (2) 174, 2011, 1429-1483.

[MV] Mateu, J., and J. Verdera: $L^{p}$ and weak $L^{1}$ estimates for the maximal Riesz transform and the maximal Beurling transform, - Math. Res. Lett. 13, 2006, 957-966.

[P] PÉREZ, C.: Weighted norm inequalities for singular integral operators. - J. London Math. Soc. 49, 1994, 296-308.

[St] Stein, E. M.: Singular integrals and differentiability properties of functions. - Princeton Univ. Press, Princeton, 1970.

Received 10 April 2014 • Accepted 29 July 2014 